\title{
BMJ Global Health Economic burden of neonatal sepsis in sub-Saharan Africa
}

\author{
Sylvia L Ranjeva, ${ }^{1,2}$ Benjamin C Warf,, ${ }^{3,4}$ Steven J Schiff ${ }^{2,5}$
}

To cite: Ranjeva SL, Warf BC, Schiff SJ. Economic burden of neonatal sepsis in subSaharan Africa. BMJ Glob Health 2018;3:e000347. doi:10.1136/ bmjgh-2017-000347

\section{Handling editor Seye Abimbola}

- Additional material is published online only. To view please visit the journal online (http://dx.doi.org/10.1136/ bmjgh-2017-000347)

Received 15 March 2017 Revised 3 October 2017 Accepted 25 October 2017

\section{(a) CrossMark}

${ }^{1}$ Medical Scientist Training Program, Pritzker School of Medicine, and Department of Ecology \& Evolution, University of Chicago, Chicago, Illinois, USA ${ }^{2}$ Department of Engineering Science and Mechanics, Center for Neural Engineering, The Pennsylvania State University, University Park, Pennsylvania, USA

${ }^{3}$ Department of Neurosurgery, Boston Children's Hospital, Boston, Massachusetts, USA ${ }^{4}$ Department of Global Health and Social Medicine, Harvard Medical School, Boston, Massachusetts, USA ${ }^{5}$ Departments of Neurosurgery and Physics, The Pennsylvania State University, University Park, Pennsylvania, USA

Correspondence to Dr Steven J Schiff; sschiff@psu.edu

\section{ABSTRACT}

Background and significance The third Sustainable Development Goal for child health, which aims to end preventable deaths of newborns and children less than 5 years of age by 2030 , cannot be met without substantial reduction of infection-specific neonatal mortality in the developing world. Neonatal infections are estimated to account for $26 \%$ of annual neonatal deaths, with mortality rates highest in sub-Saharan Africa (SSA). Reliable and comprehensive estimates of the incidence and aetiology surrounding neonatal sepsis in SSA remain incompletely available. We estimate the economic burden of neonatal sepsis in SSA.

Methods Data available through global health agencies and in the medical literature were used to determine population demographics in SSA, as well as to determine the incidence, disease burden, mortality and resulting disabilities associated with neonatal sepsis. The disabilityadjusted life years (DALY) associated with successful treatment or prevention of neonatal sepsis in SSA for 1 year were calculated. The value of a statistical life (VSL) methodology was estimated to evaluate the economic burden of untreated neonatal sepsis in SSA.

Results We conservatively estimate that 5.29-8.73 million DALYs are lost annually in SSA due to neonatal sepsis. Corresponding VSL estimates predict an annual economic burden ranging from $\$ 10$ billion to $\$ 469$ billion.

Conclusions Our results highlight and quantify the scope of the public health and economic burden posed by neonatal sepsis in SSA. We quantify the substantial potential impact of more successful treatment and prevention strategies, and we highlight the need for greater investment in strategies to characterise, diagnose, prevent and manage neonatal sepsis and its long-term sequelae in SSA.

\section{INTRODUCTION}

The third Sustainable Development Goal for child health (United Nations 2015), which aims to end preventable deaths of newborns and children under 5 years of age by 2030 , cannot be met without substantial reduction of infection-specific neonatal mortality in the developing world. Sub-Saharan Africa (SSA) bears a disproportionate burden of neonatal mortality, contributing an estimated $49.6 \%$ of under-5 deaths in 2013. ${ }^{1}$ Worldwide, neonatal infections cause an estimated $26 \%$ of under- 5 deaths, with mortality rates

\section{Key questions}

What is already known about this topic?

- Neonatal sepsis causes an estimated 750000 annual deaths worldwide with mortality highest in sub-Saharan Africa (SSA).

- Describing the economic burden of disease resulting from neonatal infections in SSA is key to advancing global public health policies.

- Neonatal sepsis is responsible for 1.6 times the global number of childhood deaths as malaria, and over four times the number of childhood deaths caused by HIV.

- Despite its disproportionate burden on childhood mortality, neonatal sepsis receives vastly less investment internationally as a public health priority compared with other major conditions.

\section{What are the new findings?}

- The incidence, disease burden, mortality and resulting disabilities associated with neonatal sepsis were estimated.

- The disability-adjusted life years (DALY) associated with successful treatment or prevention of neonatal sepsis in SSA and the value of a statistical life (VSL) methodology was estimated for the potential economic burden resulting from neonatal sepsis in SSA.

- Estimates of 5.29-8.73 million DALYs are lost annually in SSA due to neonatal sepsis, and the corresponding VSL method predicts an annual economic burden ranging from $\$ 10$ billion to \$469 billion.

\section{Recommendations for policy}

- These results highlight and quantify the scope of the public health and economic burden posed by neonatal sepsis in SSA.

- These results quantify the substantial potential impact of more successful treatment and prevention strategies, and highlight the need for greater investment in strategies to characterise, diagnose, prevent and manage neonatal sepsis and its long-term sequelae in SSA.

highest in sub-Saharan Africa. ${ }^{2-4}$ Neonatal sepsis, a condition defined as bacteraemia within the first month of life accompanied by signs of systemic infection, ${ }^{5}$ results in an estimated 750000 annual deaths worldwide. ${ }^{6-8}$ 
Describing the economic burden of disease resulting from neonatal infections in SSA is key to advancing global public health policies.

Worldwide neonatal deaths have decreased by over 3.6 million per year since 2000 , largely due to decreases in the incidence of major conditions such as pneumonia and diarrhoea. However, neonatal sepsis remains a notable counterexample to the progress in the decline of cause-specific mortality rates. ${ }^{1}$ Neonatal sepsis is responsible for 1.6 times the global number of childhood deaths as malaria, and over four times the number of childhood deaths caused by HIV. ${ }^{8}$ Despite its disproportionate burden on childhood mortality, neonatal sepsis receives substantially less investment internationally as a public health priority compared with other major conditions.

Previous estimates, reporting a range of 380000-2 000000 annual cases of neonatal sepsis in sub-Saharan Africa and 270000 annual associated deaths, ${ }^{38}$ highlight the substantial burden of disease. However, accurately estimating the burden of neonatal sepsis in sub-Saharan Africa is limited by uncertainty in the diagnosis and estimations of the incidence. Data detailing the aetiology, scope and interventions surrounding neonatal sepsis in the developing world are lacking. The identification and treatment of newborns with infection are particularly limited in the developing world. ${ }^{5}$ In resource-poor settings, with limited access to blood cultures and reliable laboratory testing, diagnosis of neonatal sepsis is typically based only on clinical indications. ${ }^{1}$ Most estimates of incidence are obtained from isolated community-based studies, where significant heterogeneity makes data difficult to compare ${ }^{8-10}$ Estimates of the incidence of neonatal sepsis in community settings in the developing world can range from 5.5/1000 live births by positive blood culture to $170 / 1000$ live births based on clinical diagnosis. ${ }^{10}$ Successful intervention in neonatal sepsis requires investment in high-quality data and reliable identification of neonatal sepsis.

The purpose of this investigation is to quantify the economic burden associated with neonatal sepsis in sub-Saharan Africa, thereby motivating investment in interventions. Using the measure of the disability-adjusted life year (DALY), we determine the years of healthy life lost and the subsequent potential economic loss that could be averted in 1 year by complete eradication of neonatal sepsis, using data from 2014.

\section{METHODS}

\section{Disability-adjusted life years}

To quantify health outcomes associated with neonatal sepsis, we calculate DALYs. The DALY measures the combined burden of premature death caused by a condition and the lifelong impact of the disease. ${ }^{11}{ }^{12}$ DALYs secondary to a particular condition are the sum of years of life lost due to premature mortality (YLL) and years lost to disability (YLD). YLDs are scaled by a disability weight (DW), a factor that ranges from 0 (perfect health) to 1 (death) to reflect the severity of the condition.

The calculation of DALYs can be moderated by age weighting and discounting. Age weighting accounts for the behavioural preference of a society with regard to age across a lifespan. ${ }^{11} 13$ Discounting, a standard practice in cost-benefit analysis, devalues future years of life relative to the present day. In disease management, discounting motivates investment in immediate healthcare expenditures relative to theoretical future benefits of investment in research and eradication programmes. ${ }^{14}$ Discounting allows DALYs calculated at each year to be summed and compared over a lifetime.

The most recent (2010) Global Burden of Disease and Risk Factors report did not use discounting or age weighting within the calculation of DALYs. ${ }^{15}{ }^{16}$ However, in the context of cost utility analysis, which provides an economic estimate of the associated burden of disease, these features may account for important cultural realities that affect the practical evaluation of disease burden. Furthermore, these features yield conservative estimates of both disease burden and economic benefits relative to calculations that do not discount or age weight. In accordance with previous analyses of the disease burden of infant conditions in $\mathrm{SSA}^{7}{ }^{7}$ we employ a standard $3 \%$ discount rate and an age weighting parameter linked to life expectancy.

To evaluate both the economic burden of disease we translate between DALY and monetary value in US\$. We use value of a statistical life (VSL) methodology ${ }^{17} 18$ to quantify the economic burden resulting from neonatal sepsis cases in sub-Saharan Africa over 1 year in 2014.

\section{Categorisation of disease processes involved with neonatal sepsis}

The long-term sequelae resulting from neonatal sepsis are difficult to quantify. We used secondary conditions with known incidence to approximate the burden caused by neonatal sepsis over a lifetime. We employed standard disability weights (DW) derived from the Global Burden of Disease and Risk Factors report ${ }^{16}$ and other literature to evaluate the impact of each secondary condition.

Eight conditions were examined in the breakdown of neonatal sepsis in SSA (figure 1): (1) death from sepsis; (2) long-term survival with postinfectious hydrocephalus (PIH), which could result in (3) childhood mortality or (4) survival with PIH with resulting neurological impairment; (5) long-term survival from sepsis with neurodevelopmental impairment, which could result in (6) cerebral palsy or (7) lifetime neurodevelopmental impairment without cerebral palsy; or (8) uncomplicated recovery following an acute 2-week period of neonatal sepsis.

The fraction of the neonatal mortality attributed to sepsis in SSA was multiplied against the current neonatal mortality rate (NMR) to determine the incidence of death secondary to neonatal sepsis. The case fatality rate associated with neonatal sepsis in the developing world was then used to determine the incidence of neonatal 


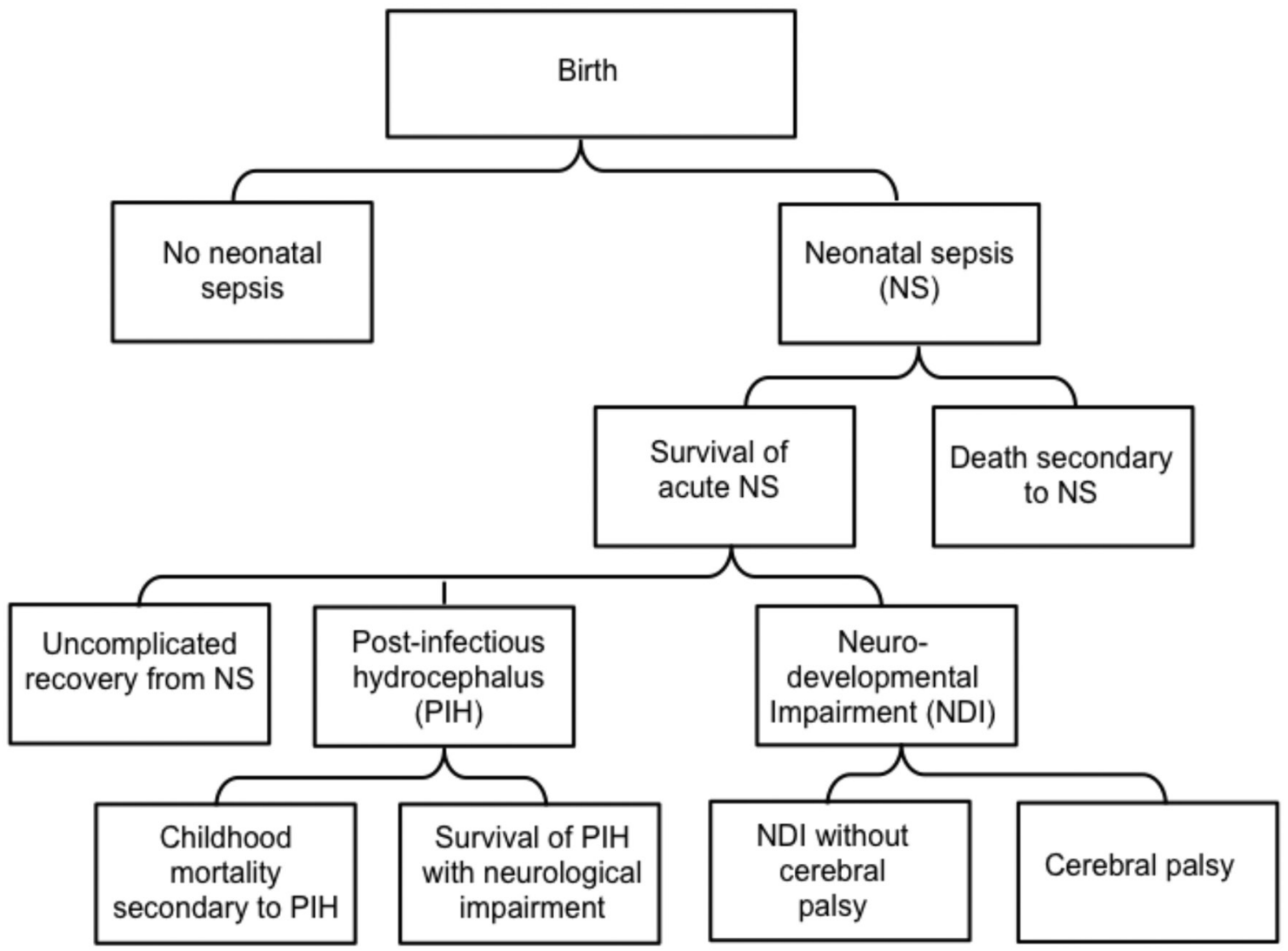

Figure 1 Flow chart of outcomes associated with neonatal sepsis in sub-Saharan Africa (SSA) used in the calculation of disability-adjusted life years (DALY). The incidence of conditions secondary to neonatal sepsis was calculated in relation to the total annual live births in SSA.

sepsis as a fraction of total annual live births (adjusted for the baseline neonatal mortality rate). Available incidence data from the literature for secondary conditions were used to determine the number of individuals in each corresponding category. Given the limited and conflicting data surrounding the epidemiology of neonatal infections in SSA, we evaluated a range of epidemiological parameters from the literature. For example, when considering the fraction of the NMR attributed to sepsis in SSA, we evaluated a range spanning the per cent NMR attributed strictly to sepsis to the per cent attributed generally to infections in the neonatal period. Table 1 lists the epidemiological parameters used for the analysis. Further discussion of the parameter choices is presented in online supplementary material.

Little data exist detailing disability outcomes that are the direct effect of neonatal sepsis in SSA. Data regarding rates of neurodevelopmental impairment and cerebral palsy following sepsis were incorporated from the literature. Hydrocephalus, a syndrome characterised by accumulation of excess cerebrospinal fluid and expansion of the cranial vault, is predominantly postinfectious following neonatal sepsis in infants in East Africa. ${ }^{19}{ }^{20} \mathrm{~A}$ previous study that examined the economic burden of disease for PIH in SSA suggests a magnitude sufficient to warrant the separate breakout for PIH that we have used here. ${ }^{7}$ The incidence of PIH in Uganda has been characterised, ${ }^{19}$ and the resulting incidence of PIH in sub-Saharan Africa was estimated using the Ugandan estimates. Furthermore, Warf $e t a l^{7}$ characterised the mortality in children less than 5 years of age from PIH after initial survival of sepsis. These rates were also extrapolated to SSA.

\section{Selection of VSL methodology for economic analysis}

Two general strategies exist to translate between DALYs and US\$: the human capital approach (HCA) and the VSL approach. The HCA was initially suggested for use with DALYs by the WHO Commission on Macroeconomics and Health. ${ }^{21}$ According to the HCA, the value of an individual to society is estimated by an individual's earnings, or their contribution to the national economy. DALYs are directly valued by the gross national income (GNI) per capita in a country or region to obtain an estimate of the potential economic burden of DALYs averted over a lifetime.

In contrast, the VSL approach is based on empirical studies of an individual's willingness to pay for incremental reductions of their risk of dying. VSL estimates may also be used to derive the value of a statistical life year (VSLY), which can be used to assign a monetary value to 1 DALY.

We suggest that VSL achieves a more realistic approximation of the economic burden associated with neonatal 
Table 1 Epidemiological parameters used in the calculation of DALYs lost secondary to neonatal sepsis in sub-Saharan Africa for the year 2014

\begin{tabular}{|c|c|c|c|}
\hline Epidemiological parameter & Value & Source & Population \\
\hline Life expectancy & 57 & World Bank ${ }^{36}$ & Sub-Saharan Africa \\
\hline Crude birth rate & $37^{*}$ & World Bank ${ }^{36}$ & Sub-Saharan Africa \\
\hline Infant mortality & $77 \dagger$ & World Bank ${ }^{36}$ & Sub-Saharan Africa \\
\hline Population & 961.5 million & World Bank ${ }^{36}$ & Sub-Saharan Africa \\
\hline NMR & $29 \dagger$ & World Bank ${ }^{36}$ & Sub-Saharan Africa \\
\hline \% NMR attributable to NS & $17 \%-29 \%$ & UN IGCME ${ }^{37}$ & Sub-Saharan Africa \\
\hline Case fatality rate for NS & $50 \%$ & WHO $2005^{338}$ & Sub-Saharan Africa \\
\hline Incidence of PIH & $3-5 \dagger$ & Warf et al ${ }^{7}$ & Ugandan infants \\
\hline $\begin{array}{l}\text { Risk of cerebral palsy secondary } \\
\text { to NS }\end{array}$ & $3.2 \%-16.3 \% \ddagger$ & $\begin{array}{l}\text { Consensus from the } \\
\text { literature§ }\end{array}$ & $\begin{array}{l}\text { International meta-analyses among } \\
\text { low birthweight infants in low-income } \\
\text { and middle-income countries }\end{array}$ \\
\hline $\begin{array}{l}\text { Risk of total neurodevelopmental } \\
\text { impairment secondary to NS }\end{array}$ & $26.4 \%-47.4 \% \S$ & $\begin{array}{l}\text { Consensus from the } \\
\text { literature§ }\end{array}$ & $\begin{array}{l}\text { International meta-analyses among } \\
\text { low birthweight infants in low-income } \\
\text { and middle-income countries }\end{array}$ \\
\hline
\end{tabular}

*Value per 1000 persons.

†Value per 1000 live births.

$\ddagger$ Range gives $95 \% \mathrm{Cl}$ reported in the literature.

$\S$ See further discussion in online supplementary materials.

DALY, daily-adjusted life year; NMR, neonatal mortality rate; NS, neonatal sepsis; PIH, postinfectious hydrocephalus; UN IGCME, United

Nations Inter-agency Group for Child Mortality Estimation.

sepsis in SSA. VSL has been sanctioned for use in cost-benefit analysis by government agencies in the USA and Australia, ${ }^{17} 22$ and is gaining ground in the international community. ${ }^{23}$ Because the methodology uses empirical evidence of human behaviour, it approximates the way in which individuals actually value reductions in health risks. For a study evaluating the economic burden of disease in the developing world, where GNI per capita averages are substantially less than those in the industrialised countries, the discrepancy between willingness to pay and lifetime income is important.

\section{Calculation of DALYs}

A detailed mathematical explanation of the calculation of DALYs is provided in the online supplementary materials. To determine the DALYs averted by the potential eradication of neonatal sepsis, we determined and summed the DALYs associated with each breakout condition attributed to sepsis (figure 1). DALYs were calculated for an initial period of acute neonatal sepsis, beginning at age zero. DALY calculations for sequential conditions were calculated from the age of onset of the condition, with the minimum age being 2 weeks, to the end of the duration of the condition. For example, in the case of PIH secondary to neonatal sepsis, we applied the DW for severe acute infection (table 2) over a 2-week period. Next, for survivors of PIH secondary to sepsis, we applied the DW for moderate intellectual disability and motor delay from age 2 weeks to the life expectancy. For the condition of childhood death following PIH secondary to sepsis, years of life lost were calculated beginning at age 5 . Assuming survival until age 5 provides the most conservative estimate of DALYs for this cohort, since we have figures for 5-year survival in this cohort. The World Bank-reported life expectancy for sub-Saharan Africa of 57 years of age was used as the upper limit of evaluation for DALYs.

\begin{tabular}{lll}
\hline \multicolumn{2}{l}{ Table 2} & DWs used in the calculation of DALYs for conditions associated with neonatal sepsis in sub-Saharan Africa. \\
\hline Disability weight & Value & Source \\
\hline DW neonatal sepsis & 0.613 & Iran Burden of Disease Study ${ }^{24}$ \\
DW moderate intellectual and motor delay & $0.221(0.141-0.314)$ & WHO Global Burden of Disease $2010^{15}$ \\
DW cerebral palsy (without intellectual disability) & 0.170 & Dutch Burden of Disease Study ${ }^{24}$ \\
DW during acute meningitis (acute PIH) & 0.894 & Dutch Burden of Disease Study ${ }^{24}$ \\
\hline
\end{tabular}

$\mathrm{PIH}$ is associated with preceding episodes of neonatal meningitis and cerebral ventriculitis. ${ }^{20}$ In order to quantify the damage to quality of life posed by $\mathrm{PIH}$, the disability weight for neonatal meningitis was used

${ }^{*}$ Range gives $95 \%$ confidence interval reported in the literature

DALY, daily-adjusted life year; DW, disability weight; PIH, postinfectious hydrocephalus. 
Table 3 Total potential DALYs averted over a lifetime, and associated economic value, given successful prevention or treatment of all cases of neonatal sepsis in sub-Saharan Africa over 1 year in 2014.

\begin{tabular}{ll}
\hline Parameter & Value \\
\hline Total DALYs & $5.29-8.73$ million \\
Economic burden & \\
$E=1.5$ & $\$ 9.93-\$ 16.40$ billion \\
$E=1$ & $\$ 58.02-\$ 95.81$ billion \\
$E=0.55$ & $\$ 284.12-\$ 469.15$ billion \\
\hline
\end{tabular}

Value ranges reflect the ranges of reported parameter values used from the literature. The economic burden is calculated using the value of a statistical life methodology under three different assumptions of income elasticity $(E=1.5, E=1, E=0.55)$

DALY, daily-adjusted life year.

The DW for each condition associated with neonatal sepsis was obtained from the 2010 Global Burden of Disease and Risk Factors study and from a 2003 study of the burden of disease in Iran. ${ }^{152425}$ The values used for each condition are given in table 2.

We used an annual discounting rate of $3 \%$ as suggested by Murray and Acharya. ${ }^{26}$ To account for age weighting in accordance with the VSL methodology, we used an age-weighting factor $\beta$ determined by empirical evidence ${ }^{27}$ and adjusted to the life expectancy reported in sub-Saharan Africa (see online supplementary material for a more detailed description of these parameters).

For several of the epidemiological parameters (tables 2 and 3), uncertainty was reported along with a point estimate of the parameter value. We report total DALYs as a range calculated over the reported intervals for each parameter.

\section{Evaluating the economic burden of neonatal sepsis: translation of DALYs to US\$ in VSL methodology}

Viscusi and Aldy presented a model to estimate the value of a statistical life in US\$ in countries where formal studies have not been conducted to determine a base estimate of VSL. ${ }^{18}$ The Department of Transportation (DOT) estimates a base VSL of $\$ 9.2$ million for the USA based on empirical studies. ${ }^{22}$ The VSL of a country in SSA can be determined comparatively as follows:

$$
V S L_{S S A}=V S L_{U S A} *\left(\text { Income }_{S S A} / \text { Income }_{U S A}\right)^{E}
$$

where $V S L_{S S A}$ is the value of a statistical life for sub-Saharan Africa, Income $S S A$ is the gross national income (GNI) per capita for sub-Saharan Africa, VSL $L_{U S A}$ is the value of a statistical life for the USA, Income $_{U S A}$ is the GNI per capita in the USA, and $E$ denotes the income elasticity of VSL. ${ }^{28}$

In VSL methods, the income elasticity moderates the extrapolation of VSL estimates across countries based on differences in GNI per capita. Constant VSL $(E=0)$ implies that citizens in a country in SSA and citizens in the USA would be willing to pay equally for an incremental decrease in risk of death or disability. However, that amount would represent a vastly larger percentage of total income for the citizens of a country in SSA than for those in the USA. If VSL varies proportionally to income $(E=1)$, then the value of a statistical life of a person in SSA would appear lower than that of a person in the USA. If mortality risk reductions are viewed as luxuries $(E>1)$, then it follows that a person in SSA, with higher economic constraints, would not be as willing to pay an equivalent relative amount for risk reduction, resulting in a further lowering of VSL. However, if mortality risk reductions are viewed as necessities $(E<1)$, then equation (1) can be used to translate VSL across income differences. Viscusi and Aldy suggest an elasticity of $E=0.55,{ }^{18}$ which reflects the willingness to pay rather than actual income in the calculation of VSL for a country in sub-Saharan Africa. Hammit and Robinson ${ }^{28}$ argue, however, that the income elasticity of VSL is greater than 1.0 for poor countries, and recommend against using income elasticity values below 1 . We therefore used two other scenarios $(E=1$, direct GNI scaling, and $E=1.5$, luxury GNI scaling) to provide a more complete spectrum of economic estimates.

In order to use VSL to evaluate the economic impact of DALYs associated with each condition, we calculated an age-independent annual value of a statistical life lost per year, or the VSLY, in sub-Saharan Africa and multiplied this value by the number of DALYs associated with each condition.

\section{RESULTS}

For 2014, based on table 1, we conservatively estimate 355500-605750 annual cases and 177500-302870 annual deaths due to neonatal sepsis in SSA.

The lifelong burden of neonatal sepsis was categorised based on secondary long-term sequelae (figure 1), each with a unique disability weight (DW) according to the Global Burden of Disease and Risk Analysis report and a 2003 study conducted in Iran (table 2). ${ }^{152425}$ If all cases of neonatal sepsis for 1 year had been successfully treated or prevented, a total of 5.29-8.73 million DALYs could have been averted over an average lifetime (table 3).

The annual economic implications of the potential DALYs averted by successful treatment or prevention of sepsis are substantial. The VSL approach for economic valuation of DALYs suggested a wide range of potential value from $\$ 9.3$ billion $(E=1.5)$ to $\$ 469.15$ billion $(E=0.55)$. The economic impact of each scenario is provided in table 3 .

\section{DISCUSSION}

This work quantifies the substantial disease burden posed by neonatal sepsis in SSA, and is to our knowledge the first to provide an economic estimate of the burden of 
this condition for the region. Our results indicate that neonatal sepsis presents a \$10-\$469billion financial burden that could be alleviated through successful treatment and prevention.

Though large in scope, our results likely underestimate disease burden. Previous studies estimated 1-2 million yearly cases of neonatal sepsis in SSA. ${ }^{3}$ Recent literature provided a more conservative range of 380000-870000 annual cases, and 270000 annual deaths due to neonatal sepsis for the year $2010 .{ }^{8}$ Our estimates of annual incidence and mortality secondary to neonatal sepsis in SSA provide a conservative range that overlaps these estimates. Furthermore, our estimates of the DALYs secondary to neonatal sepsis in sub-Saharan Africa are substantially lower than the estimated 11-30 million DALYs reported in the 2010 Global Burden of Disease study. ${ }^{16}$ This difference is partly due to our choice to use age weighting and discounting in our calculation of DALYs. We therefore maintain a conservative estimate of the corresponding economic impact.

The broad estimated range of the economic burden reflects different interpretations of the income elasticity between the USA and SSA. We prefer to not impose value judgements on populations regarding the value of life biased towards necessity versus luxury, but offer the full range of quantitative estimates in current use. Within this range lie the applicable values, and they reflect the enormous burden of neonatal sepsis on morbidity, mortality and economic burden.

Broadly, high-quality data are needed to make policy decisions that can help reduce neonatal mortality. Neonatal sepsis has begun to gain recognition in the international community as a major and under-represented global health crisis, ${ }^{529}$ but information regarding the aetiology, scope and treatment strategies remains substantially underdeveloped. Research into the complete spectrum of invasive microorganisms contributing to neonatal sepsis in sub-Saharan Africa-the neonatal septisome $e^{3031}$-is necessary both to optimise treatment and to develop rational public health strategies. Through thorough and standardised methodology and access to accurate diagnosis, the incidence of sepsis can be better understood across communities. Furthermore, to accurately evaluate the burden of sepsis, better data are needed detailing the scope, severity and duration of long-term sequelae. For example, while the burden of an acute febrile episode can be approximated over a very short (weeks) timespan by the disability weight for acute meningitis, data are lacking to estimate more accurately the subsequent impairment over a lifetime, and the effect on shortening that lifetime.

Investments in public health efforts to counter the substantial burden of neonatal sepsis are important at various population scales. We quantified the burden of neonatal sepsis at the regional level across sub-Saharan Africa, in line with the focus under the Sustainable Development Goals on reducing disparities in child survival between SSA and other world regions. ${ }^{32}$
Such regional-level analysis puts our work in context with important contemporary estimates of the burden of childhood and neonatal disease at the same scale. ${ }^{18}$ However, country-specific data about the incidence and outcomes of neonatal sepsis could be gained through investments at the national level across SSA. Our model could be used to quantify the country-specific burden of neonatal sepsis, informing public health policy and decision-making at the individual national scale.

Serious neonatal infections bear a substantial morbidity risk even when mortality is averted through successful treatment, and the optimal treatment for these infants is prevention. More adequate prevention poses a substantial challenge, since mounting the effort to identify regionally the most common routes of infection, and the potential role of multiorganism primary infections or sequential secondary infections, remains a substantial barrier towards rational and granular public health preventative policies. Recent evidence suggests that rainfall and climate may play a significant role in neonatal sepsis cases leading to nervous system involvement in Uganda ${ }^{33}$ and the role that climate plays in neonatal sepsis in SSA remains to be defined.

The economic impact of neonatal mortality is connected to a more complex set of social and economic issues in a society than is reflected in our focus on individual DALY and VSL considerations. For instance, in the economic report Uganda $2040,{ }^{34}$ the high birth rate (averaging 6.7 per woman per lifetime) is considered as a major economic burden on society. At such high birth rates, women are effectively excluded from the economy. High neonatal mortality helps drive such high birth rates. If infant mortality is decreased, the ripple effect through the society can be economically substantial.

We are mindful of the limitations associated with the VSL methodology used to evaluate the economic burden associated with neonatal sepsis.

Of the global infectious killers of children, neonatal sepsis may be the most treatable and preventable. We are cautious in this statement, because without a more comprehensive assessment of the spectrum of microorganisms and their routes of infection in the developing world, the magnitude of the challenge posed to reduce the disability, death and economic impact of this condition remains uncertain. Our findings help quantify the immense burden posed by neonatal sepsis in SSA, and stress that the achievement of international milestones in child health will require a refocusing of priorities that includes more comprehensive characterisation of neonatal sepsis to serve as a basis for more effective treatment and rational public health policy.

\section{Limitations of the study}

Our estimates were primarily limited by the quality of comprehensive and accurate information available for neonatal sepsis worldwide. The debilitating outcomes of this syndrome remain poorly characterised in the 
developing world. Meningitis, PIH and neurodevelopmental impairment with possible cerebral palsy were the best defined disabilities in survivors of neonatal sepsis because they are quantifiable through clinical examination. Information regarding disabilities affecting other major organ systems (kidney, lung, liver, and so on) that arise from neonatal sepsis and impair health in survivors is generally unavailable. The inclusion of accurate disability figures related to other organ systems would refine our economic burden estimates.

The use of the value of a statistical life framework for the economic valuation of the burden of neonatal sepsis in SSA carries several limitations. Notably, the use of GNI per capita as a uniform marker of individual economic potential ignores important cultural heterogeneities. Our results must therefore be interpreted with caution. To partially address the associated uncertainty in the translation of DALYs to US\$, we estimated the economic burden of disease across a range of potential income elasticities, and we avoided focusing on a measure of central tendency (eg, mean).

Because we were limited by lack of available incidence data describing the sequelae of neonatal sepsis in SSA, we at times needed to employ available incidence data from other areas of the developing world. The literature describing conditions secondary to neonatal sepsis in the developing world relies heavily on compilations of isolated community studies, which limits its generalisability to SSA. ${ }^{5}$ The incidence of PIH was extrapolated to SSA from estimates of the PIH burden in Uganda, ${ }^{7}$ and the outcomes of PIH were conservatively estimated assuming all children were optimally treated. ${ }^{35}$

Despite these uncertainties, our estimates, though substantial, likely underestimate the true burden of neonatal sepsis in sub-Saharan Africa. Our estimates of the incidence, DALYs and, therefore, the economic burden attributable to neonatal sepsis are conservative with respect to current estimates from the literature.

\section{CONCLUSION}

Neonatal sepsis poses a massive public health burden for sub-Saharan Africa, with significant associated economic consequences. Strategic investment in characterisation of the disease aetiology, diagnosis, treatment and prevention is warranted.

Acknowledgements We are grateful for the helpful discussions with W Kisamba Mugerwa, Chairperson of the National Planning Authority of Uganda. We appreciate the helpful comments on our manuscript by B Alkire, J Vincent and J Meara.

Contributors Substantial contributions to the conception or design of the work; or the acquisition, analysis or interpretation of data for the work: SJS, BCW, SLR. Drafting the work or revising it critically for important intellectual content: SJS, BCW, SLR. Final approval of the version to be published: SJS, BCW, SLR. Agreement to be accountable for all aspects of the work in ensuring that questions related to the accuracy or integrity of any part of the work are appropriately investigated and resolved: SJS, BCW, SLR.

Funding This work received the support of the Penn State University endowment funds of Harvey F Brush, and NIH Director's Pioneer Award 5DP1HD086071 (SJS).

Competing interests None declared.
Provenance and peer review Not commissioned; externally peer reviewed.

Open Access This is an Open Access article distributed in accordance with the Creative Commons Attribution Non Commercial (CC BY-NC 4.0) license, which permits others to distribute, remix, adapt, build upon this work non-commercially, and license their derivative works on different terms, provided the original work is properly cited and the use is non-commercial. See: http://creativecommons.org/ licenses/by-nc/4.0/

(C) Article author(s) (or their employer(s) unless otherwise stated in the text of the article) 2018. All rights reserved. No commercial use is permitted unless otherwise expressly granted.

\section{REFERENCES}

1. Liu L, Oza S, Hogan D, et al. Global, regional, and national causes of child mortality in 2000-13, with projections to inform post-2015 priorities: an updated systematic analysis. Lancet 2015;385:430-40.

2. Lawn JE, Cousens S, Zupan J, et al. 4 million neonatal deaths: when? where? why? The Lancet 2005;365:891-900.

3. Vergnano S, Sharland M, Kazembe P, et al. Neonatal sepsis: an international perspective. Arch Dis Child Fetal Neonatal Ed 2005;90:F220-f224.

4. UNICEF. The state of the world's children 2009: maternal and newborn health. New York: Unicef, 2008.

5. Qazi SA, Stoll BJ. Neonatal sepsis: a major global public health challenge. Pediatr Infect Dis J 2009;28:S1-S2.

6. Watson RS, Carcillo JA. Scope and epidemiology of pediatric sepsis Pediatr Crit Care Med 2005;6:S3-S5.

7. Warf BC, Alkire BC, Bhai S, et al. Costs and benefits of neurosurgical intervention for infant hydrocephalus in sub-Saharan Africa. $J$ Neurosurg Pediatr 2011;8:509-21.

8. Seale AC, Blencowe $\mathrm{H}$, Zaidi A, et al. Neonatal severe bacterial infection impairment estimates in South Asia, sub-Saharan Africa, and Latin America for 2010. Pediatr Res 2013;74:73-85.

9. Thaver D, Ali SA, Zaidi AK. Antimicrobial resistance among neonatal pathogens in developing countries. Pediatr Infect Dis $J$ 2009;28:S19-S21.

10. Thaver D, Zaidi AK. Burden of neonatal infections in developing countries: a review of evidence from community-based studies. Pediatr Infect Dis J 2009;28:S3-S9.

11. Murray CJ. Quantifying the burden of disease: the technical basis for disability-adjusted life years. Bull World Health Organ 1994;72:429.

12. Mundial B. World development report 1993; investing in health. UK Oxford University Press, 1993

13. Hammitt JK, Haninger K. Valuing fatal risks to children and adults: effects of disease, latency, and risk aversion. J Risk Uncertain 2010;40:57-83.

14. Viscusi WK. Discounting health effects for medical decisions. Valuing health care 1995;7.

15. Lopez A, Mathers C, Ezzati M, et al. Global burden of disease and risk factors. New York: Oxford University Press, The World Bank, 2006.

16. Murray CJ, Vos T, Lozano R, et al. Disability-adjusted life years (DALYs) for 291 diseases and injuries in 21 regions, 1990-2010: a systematic analysis for the Global Burden of Disease Study 2010. Lancet 2012;380:2197-223.

17 Economics A. The health of nations: the value of a statistical life. Report for Office of the Australian Safety and Compensation Council, 2008.

18. Viscusi WK, Aldy JE. The value of a statistical life: a critical review of market estimates throughout the world. J Risk Uncertain 2003;27:5-76.

19. Warf BC, Dagi AR, Kaaya BN, et al. Five-year survival and outcome of treatment for postinfectious hydrocephalus in Ugandan infants. J Neurosurg Pediatr 2011;8:502-8.

20. Warf BC. Hydrocephalus in Uganda: the predominance of infectious origin and primary management with endoscopic third ventriculostomy. J Neurosurg 2005;102:1-15.

21. World Health Organization. Macroeconomics and health : investing in health for economic development : executive summary / report of the Commission on Macroeconomics and Health. Geneva: JD Sachs to Gro Harlem Brundtland, Director-General of the WHO (20 December), WHO, 2001.

22. Rogoff P, Thomson K. Guidance to treatment of the economic value of a statistical life (VSL) in US Department of Transportation Analyses: 2014 adjustments, 2014.

23. Alkire B, Hughes CD, Nash K, et al. Potential economic benefit of cleft lip and palate repair in sub-Saharan Africa. World J Surg 2011;35:1194-201. 
24. Naghavi M, Abolhassani F, Pourmalek F, et al. The burden of disease and injury in Iran 2003. Popul Health Metr 2009;7:1.

25. Mathers C, Vos T, Stevenson C. The burden of disease and injury in Australia. Australia: Australian Institute of Health and Welfare, 1999.

26. Murray CJL, Acharya AK. Understanding DALYs. J Health Econ 1997;16:703-30.

27. Aldy JE, Viscusi WK. Adjusting the value of a statistical life for age and cohort effects. Rev Econ Stat 2008;90:573-81.

28. Hammitt JK, Robinson LA. The income elasticity of the value per statistical life: transferring estimates between high and low income populations. J Benefit Cost Anal 2011;2:1-29.

29. US Government Printing Office. Hydrocephalus teatment in Uganda: leading the way to help children : hearing before the subcommittee on Africa, global health, and human rights of the committee on foreign affairs, house of representatives, one hundred twelfth congress. USA: US Government Printing Office, 2011.

30. Kiwanuka J, Bazira J, Mwanga J, et al. The microbial spectrum of neonatal sepsis in Uganda: recovery of culturable bacteria in mother-infant pairs. PLoS One 2013;8:e72775.

31. Schiff SJ, Kiwanuka J, Riggio G, et al. Separating putative pathogens from background contamination with principal orthogonal decomposition: evidence for leptospira in the ugandan neonatal septisome. Front Med 2016;3.

32. Liu L, Oza S, Hogan D, et al. Global, regional, and national causes of under-5 mortality in 2000-15: an updated systematic analysis with implications for the sustainable development goals. Lancet 2016;388:3027-35

33. Schiff SJ, Ranjeva SL, Sauer TD, et al. Rainfall drives hydrocephalus in East Africa. J Neurosurg Pediatr 2012;10:161-7.

34. National Planning Authority U. Uganda vision 2040, 2013. http://npa ug/uganda-vision-2040/ (accessed on 9 Oct 2016).

35. Kulkarni AV, Schiff SJ, Mbabazi-Kabachelor E, et al. Endoscopic treatment versus shunting for infant hydrocephalus in Uganda. $N$ Engl J Med 2017;377:2456-64.

36. The World Bank. World development indicators. Washington DC, USA: The World Bank, 2014

37. World Health Organization. Levels and trends in child mortality: report 2015: estimates developed by the UN inter-agency group for child mortality estimation. New York: United Nations Children's Fund, 2015.

38. Hamer DH. Public health and infectious diseases. Netherlands: Elsevier, 2010. 